\title{
QED nonlinear effects in photonuclear processes
}

\section{V.G.Nedorezov ${ }^{1}$}

Institute for Nuclear Research RAS

117312 Prospect of 60 let Oktabra, 7A, Moscow, Russia

E-mail:vladimir@cpc.inr.ac.ru

\section{Abstract}

Experimental data related to the nonlinear QED effects in photonuclear processes are presented. This includes above all inclusive cross sections for photo - and electrofission of actinide nuclei at intermediate energies. For comparison, the Coulomb dissociation of relativistic nuclei effects are discussed. New photo-nuclear experiments conducted by femtosecond terawatt laser are described and discussed in frame of the presented topic. 


\section{Introduction}

Nonlinear QED effects in the photon scattering in the Coulomb field were mentioned at first in 1937 when Delbruck studied this process which was explained by the virtual electron - positron pair production due to the vacuum polarization (see Diagram 1 in Figure 1). This process in modern terminology is described now in textbooks on quantum electrodynamics (see, for example [1]). Now a common feature of nonlinear effects is the large number of vertices (more than two) in the Feynman diagrams for the processes under study. For example, diagram 2 in Figure 1. shows the splitting of the photon, which is experimentally was studied in [2]. Diagram 3 is related to the multiphoton exchanges that dominate in the Coulomb interaction of relativistic nuclei (see eg, [3]). Last diagram is in principle valid for any charged particles, but in the case of electron scattering the multiphoton exchange has not yet been addressed.

All the above processes are long-range, as occur in the Coulomb field of the nucleus. However, in this paper we pay attention only on those that are accompanied by the excitation of nuclei. This allows a new look at the problem of describing the inclusive cross sections for electron scattering on heavy nuclei. Interest in this work also is stimulated by the new data on photonuclear reactions obtained with a beam of femtosecond lasers (see, eg, [4]), which are also considered to be related to nonlinear effects in the QED processes. These reactions are fundamentally different from the classical ones that they come under the influence of the superposition of a huge number of waves (up to $10^{20}$ ), where the wavelength is close to the visible range while the energy of the wave packet increases to the nuclear scale (MeV or even $\mathrm{GeV}$ ). Thus, the classical relation between the energy of a photon and its wavelength is not valid. Mechanism of interaction of these waves with the nuclei are still poorly understood. In this paper we attempt to combine different on the first glance experiments within the general problem of nonlinear effects of QED in photonuclear processes.

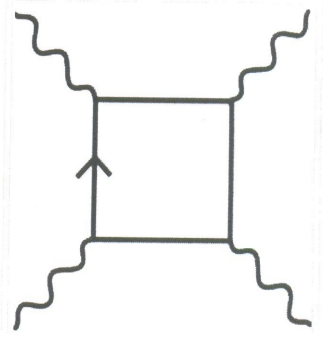

1

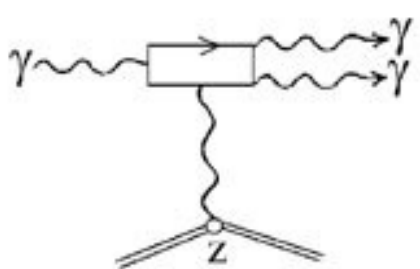

2

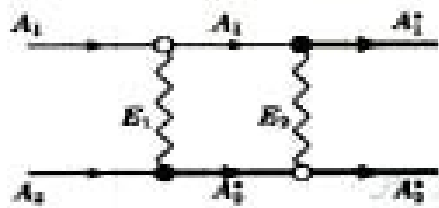

3

Fig.1.

Feynman diagrams for nonlinear effects:

1 - Delbruck scattering of a photon by the Coulomb field of the nucleus,

2 - the splitting of the photon,

3 - Coulomb interaction of relativistic charged particles. 


\subsection{Electro - and Photofission of Actinide Nuclei}

Inclusive experiments on electro - and photofission of actinide nuclei at low and medium energies (from threshold to several $\mathrm{GeV}$ ) were performed in the 80-ies in the INR [5], as well as in cooperarion with BINP [6] and KIPT [7]. According to these data there were published reviews $[8,9]$ and the monograph [10]. These works were at that time considered the completion stage in the study of electromagnetic interactions of heavy nuclei; small (about 10\%) deviations from the model Weizsecker - Williams [11] in the single-photon exchange approximation were interpreted as phenomelogical corrections due to the distortion of the plane wave in the Coulomb field of the nucleus, or the finite size of the nucleus.

The yield of the inclusive reaction of electron scattering (in the simplest case of onephoton exchange) in frame of the Weizecker - Williams model is described by the spectrum of virtual photons as follows:

$$
\Delta \mathrm{Y}\left(\mathrm{E}_{\mathrm{e}}, \mathrm{E}_{\gamma}\right)=\mathrm{N}^{\lambda 1}\left(\mathrm{E}_{\mathrm{e}}, \mathrm{E}_{\gamma}\right) * \mathrm{~N}_{\mathrm{t}} * \sigma\left(\mathrm{E}_{\gamma}\right) \Delta \mathrm{E}_{\gamma}
$$

where $\sigma(\mathrm{E} \gamma)$ - cross-section, depending on the energy of the virtual photon,

$\mathrm{N}_{\mathrm{t}}$ - the number of target nuclei, $\mathrm{N}^{\lambda 1}\left(\mathrm{E}_{\mathrm{e}}, \mathrm{E}_{\gamma}\right)$ - spectrum of virtual photons, as shown in Figure 2 and described by formula 2 [10])

$$
N^{\lambda I}\left(E_{e}=E_{e}, E_{\gamma}\right)=\frac{\alpha}{\pi}\left\{\left[1+\left(\frac{E_{e}-E_{\gamma}}{E_{e}}\right)^{2}\right] x \ln \frac{2 E_{e}\left(E_{e}-m_{e}\right)}{m_{e} E_{e}}-C_{L}\right\}
$$

Fig.2

Spectrum of virtual photons in electron scattering with an energy $E_{e}$ of $100 \mathrm{MeV}$ at the nucleus of uranium [10]

$$
\mathrm{N}^{\lambda 1}\left(\mathrm{E}_{\mathrm{e}}, \mathrm{E}_{\gamma}\right)
$$

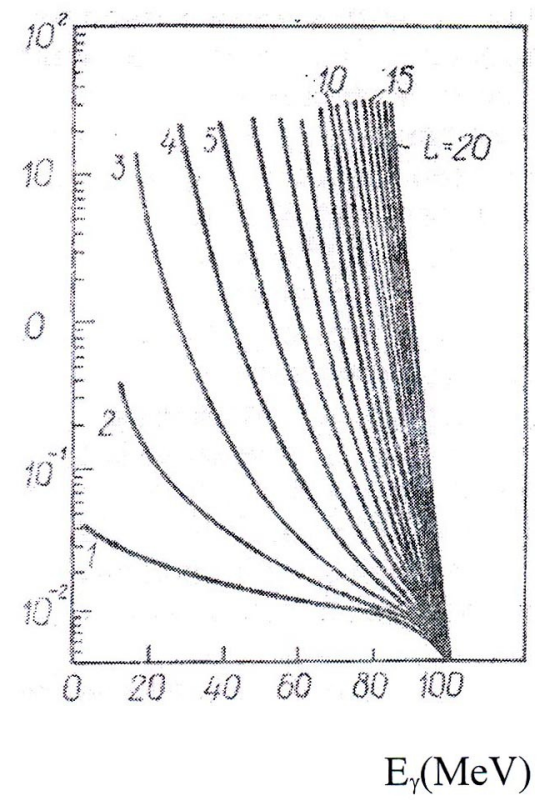


This spectrum depends on the multipolarity $\lambda 1$ and structure functions :

$\mathrm{C}_{\mathrm{L}}=2\left(\mathrm{E}_{\mathrm{e}}-\mathrm{E}_{\gamma}\right) / \mathrm{E}_{\mathrm{e}}$ for $\lambda \mathrm{l}=\mathrm{E} 1, \mathrm{C}_{\mathrm{L}}=0$ for $\lambda \mathrm{l}=\mathrm{M} 1, \mathrm{C}_{\mathrm{L}}=8 / 3\left[\left(\mathrm{E}_{\mathrm{e}}-\mathrm{E}_{\gamma}\right) / \mathrm{E}_{\mathrm{e}}\right] / 2$ for $\lambda \mathrm{l}=\mathrm{E} 2$, $\mathrm{C}_{\mathrm{L}}$ depends on the size of the nucleus and its charge. In general, one should consider a separate contribution to the transverse and longitudinal components, to correct for the distortion of the wave in the nuclear field, etc. All of this, given the strong dependence on the multipolarity, makes the analysis inconclusive. Therefore, experimental determination of the spectra of virtual photons is an actual problem until now.

However, some qualitative effects in electro - and photofission processes can be interpreted now as nonlinear QED effects contribution, probably. For example, Figure 3 shows the cross section of the spontaneously fissioning isomer production for Am nuclei, which can be related unexpectedly to a very low excitation energies (near the fission barrier) in spite of the high electron energy of $1 \mathrm{GeV}$.

Fig.3

Cross section of the reaction ${ }^{243} \mathrm{Am}(e, n){ }^{242 m f} \mathrm{Am}$.

Points shows an experimental result, dotted line is the result of approximation. The solid curve shows the contribution of the dipole resonance (with errors) [7].

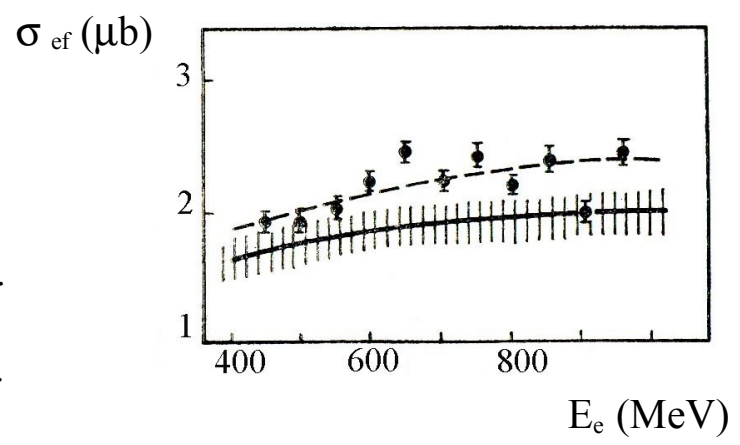

One can see that the yield of the spontaneously fissioning isomer exceeds the $30 \%$ contribution relatively to the giant dipole resonance one. It is known that spontaneously fissioning isomers are related to the existence double humped fission barrier, which disappears at the excitation energy above about $50 \mathrm{MeV}$. In this case the excitation of the isomer can be explained by the process with small energy transfer, which is a nonlinear effect of QED for the photonuclear reaction.

Another qualitative effect which could not be expalinned using a traditional knowledge was also found in the photofission of actinide nuclei in the nucleon resonance energy region (see Fig.4).

These results were first obtained in the beam of back-scattered Compton photons in BINP, [12] and later confirmed in JLAB [12]. We see that photofission cross sections for these nuclei is about $20 \%$ higher than the total photoabsorption cross section, calculated as the sum of the cross sections of meson photoproduction on quasi-free nucleons ("universal curve"). They pointed to the possible contribution of additional mechanisms of absorption of photons by nuclei to the mechanism associated with the meson photoproduction on nucleons. 

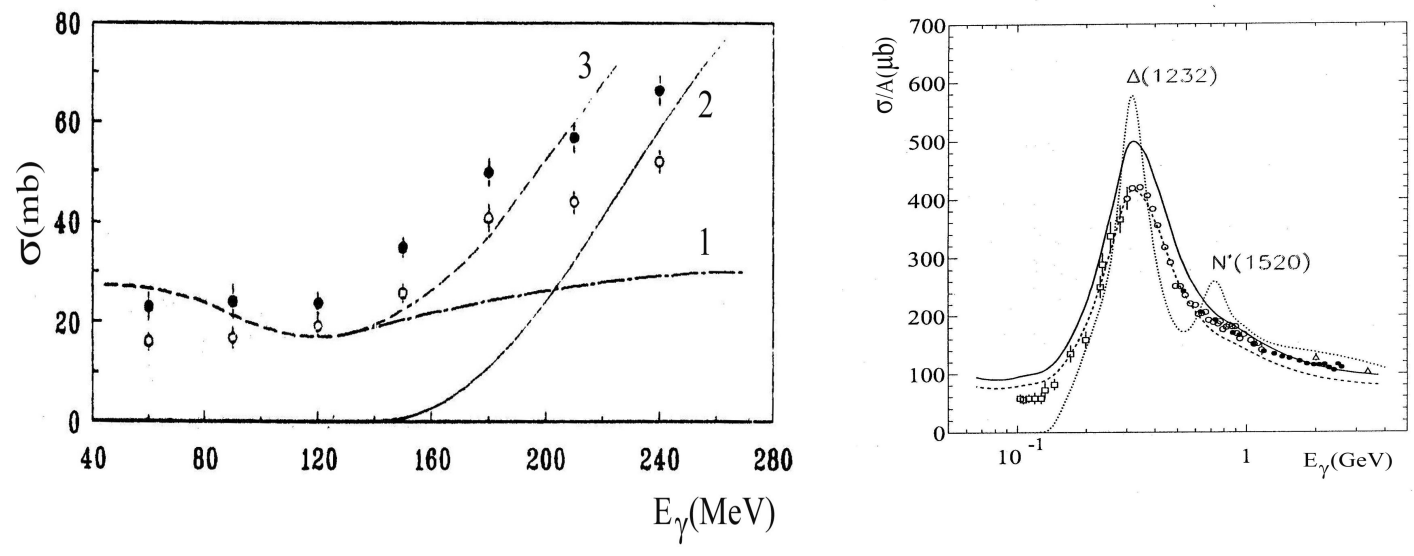

Fig 4

Left figure : photofission cros section for ${ }^{238} \mathrm{U}$ and ${ }^{237} \mathrm{~Np}$ (open and solid points respectively). Curves 1-3 represent the result of model calculation [11].

Right figure : total photoabsorption cross section for actinide nuclei (full curve), circles and dotted line represent the universal curve, points correspond to the free proton [12].

In [14] it was shown that for the photon energy range from 60 to $240 \mathrm{MeV}$, the ratio of the symmetric mode to the asymmetric one (S/A) is on average about $40 \%$ for uranium nuclei. This result means that nuclear fission after absorbing a photon comes from the relatively low excited states. To explain this fact one can suggest two options: or nucleus fission occures after the intranuclear cascade, when most of the energy is carried away by the cascade particles, either we have an example of the low energy transfer process. To check this suggestion in [15] there was measured the probability of reaction $(\gamma, \mathrm{xf})$, where $\mathrm{x}$ is a particle emitted in forward direction in coincidence with fission fragments. The result of these measurements is presented in Figure 5.

Fig. 5

The ratio of the reaction probability $(\gamma, x f)$, where $x$ particles emitted forward in coincidence with fission fragments, to the total photofission ${ }^{238} \mathrm{U}$ cross section [15].

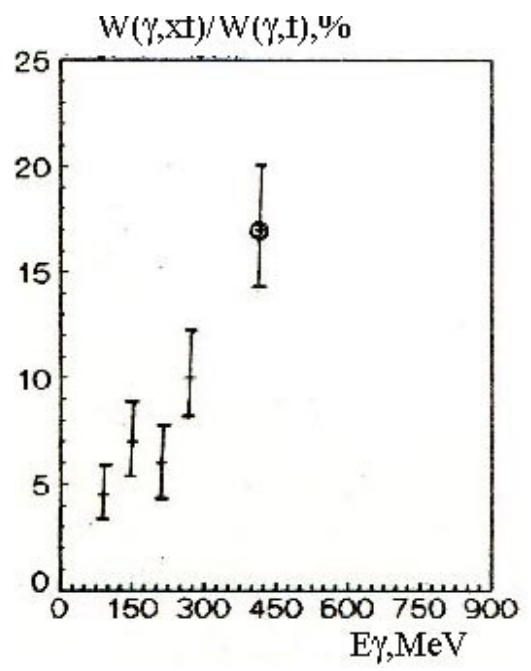


The results mentioned above indicate, probably, that in the study of reactions with intermediate energy photons (real or virtual) one need to take in account additional, more complex mechanisms which were not taken earlier into account. Such mechanisms correspond to the large number of vertices in Feinman diagrams. In modern terms, this means that the role of nonlinear effects of QED can be noticeable and this item requires both experimental and theoretical study.

\subsection{Femtosecond Laser Photonuclear Experiments}

The interaction of femtosecond laser pulses with matter also may be considered as an example of nonlinear effects of QED in photonuclear processes. In this case the wave packet is the result of interference (addition) of a huge number of individual waves.

Parameters of laser systems, which exist in the world, are the following (see eg the review [4]). The pulse duration is close to $10^{-15} \mathrm{~s}$ (femtoseconds), the length of the wave packet is less than $10 \mu \mathrm{m}$ (10 wavelengths), pulse energy up to $100 \mathrm{~J}$, power up to $10^{15} \mathrm{~W}$ (petawatt). Radiation is focused to a spot radius of about $10 \mu \mathrm{m}$, which ensures $\mathrm{W}=10^{20} \mathrm{~W} / \mathrm{cm}^{2}$ ). In this case, the electric field $\mathrm{E}=10^{12} \mathrm{~V} / \mathrm{sm}$. For comparison, in the hydrogen atom, $\mathrm{E}=10^{9} \mathrm{~V} / \mathrm{cm}$., The breakdown of mica coresponds to $10^{6} \mathrm{~V} / \mathrm{cm}$. At $\mathrm{E} \sim$ $10^{11} \mathrm{~V} / \mathrm{sm}$, respectively $\mathrm{W} \sim 10^{18} \mathrm{~W} / \mathrm{cm}^{2}(\lambda=1 \mu \mathrm{m})$ electrons are accelerated to relativistic speeds closely to the speed of light. Therefore, such field is called as relativistic one.

At present, as seen in Fig.6, taken from [4], the electron acceleration level is avalable now up to $1 \mathrm{GeV}$.

\section{Fig.6}

Increase in the power of femtosecond lasers in recent years. [4]

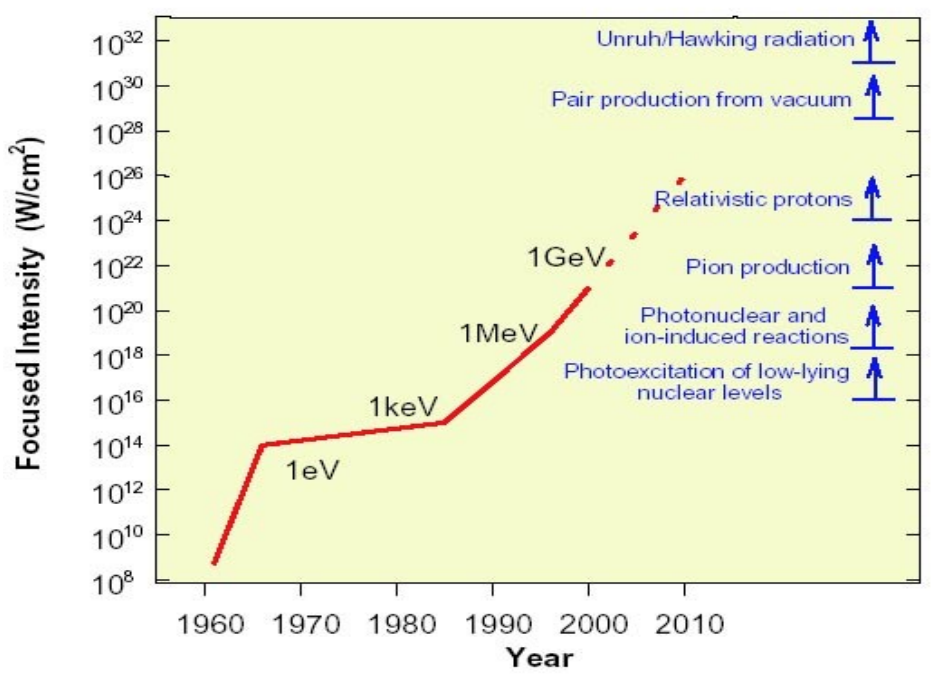

Scheme of the experiment performed in ILC MSU in collaboration with INR [15] is presented in Figure 7. 
$\lambda=800 \mathrm{~nm}$,

$\mathrm{t}=50 \mathrm{fs}$,

$\mathrm{f}=10 \mathrm{~Hz}$,

$\mathrm{E}=50 \mathrm{MJ}$,

$\mathrm{D}=4 \mu \mathrm{m}$.

$\mathrm{P}=10^{19} \mathrm{Wt} / \mathrm{cm}^{2}$

respectively Ee $\sim 1 \mathrm{MэB}$.

Target $-\mathrm{Pb}$

Detector $\Delta \mathrm{E}-\mathrm{E}$ :

$6 \mathrm{~mm}$ plastic scintillator

NE102

$\mathrm{NaI} 65 \times 65 \mathrm{~mm}$

PMT FEU-143

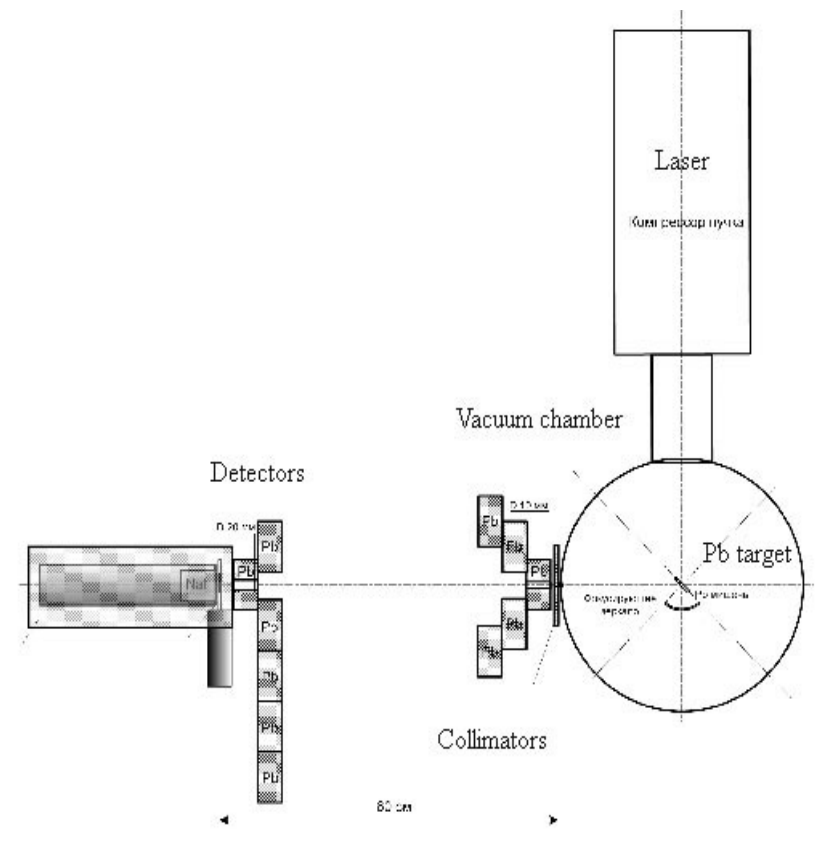

Fig. 7

Laser photonuclear installation ILC MSU and its parameters.

Preliminary experimental results obtained with a scintillation spectrometer $\Delta \mathrm{E}-$ E, are shown in Fig. 8.
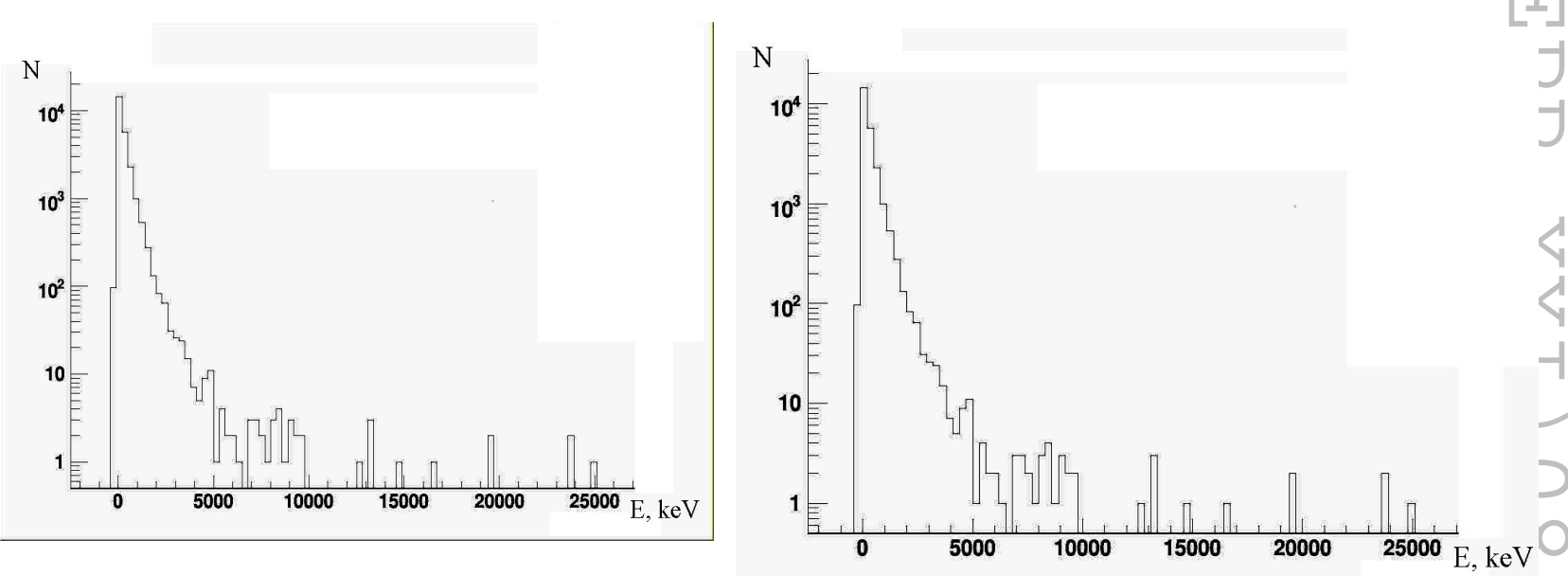

Fig.8

Spectra of electrons and photons from $\mathrm{Pb}$ target, measured by plastic $\Delta E$ (left) and $\mathrm{NaJ}(\mathrm{Tl})$ detector [15]. 
We see that the emission from the lead target is consist of electrons and photons with a maximum energy of about $5 \mathrm{MeV}$. In this case, both spectra are exponential in nature, but in the spectrum of $\Delta \mathrm{E}$ one can distinguish two exponents. Here, both electrons and photons are involved in the total output, and we could not separate them clearly yet.

The results indicate that under given conditions photonuclear processes induced by the femtosecond laser pulses have a two step nature: at first lectrons are accelerated and then they produce the bremsstrahlung photons. Then these photons interact with nuclei. Direct excitation of the nuclei by the long wave photons is not observed. To understand the nature of photonuclear interactions in detailes, the new theoretical and experimental studies are required.

\section{Acknowledgments} discussions.

The author thanks Drs. A.L'vov, P.Zarubin, I.Pshenichnov for useful

Work is supported by RFBR, grant 11-02-00286

\section{References}

[1] Ahiezer A.I., Berestetsky V.B, Quantum electrdynamics, Moscow, Nauka, (1981).

[2] Sh.Zh.Akhmadaliev e.a. Phys.Rev. C 58 (1998) 2844.

[3] I.A.Pshenichnov, Proc.of X Unt. Seminar EMIN-2009, Moscow, ed.by G.M.Gurevich, (2003), 234-242.

[4] A.B.Andreev, B.M.Gordienko, A.B.Savel'ev. Quantum electronics 31,11. (2001), 941-956

[5] V.L.Kuznetsov, L.E.Lazareva, V.G.Nedorezov e.a. Nucl.Phys. A324 (1979) 29.

[6] V.L.Kuznetsov, L.E.Lazareva, V.G.Nedorezov e.a. Nucl.Phys. A381 (1982) 1439.

[7] V.L.Kuznetsov, L.E.Lazareva, V.G.Nedorezov e.a . JETF Lett. 40, 10 (1984) 445.

[8] V.G.Nedorezov, Yu..N.Ranyuk. Phys.of elementary partiles, JINR 2 (1984) 379.

[9] V.G.Nedorezov, A.A.Turing, Yu.M.Shatunov. Uspekhi of Phys.Nauk. 174, 4 (2004) 353.

[10] V.G.Nedorezov, Yu..N.Ranyuk, Photofission above the giant resonance. "Naukova dumka», ed.by P.A.Klucharev, (1989), Kiev.

[11] C.F. Weizsacker, Z.Phys. 29 (1934) 612-625, E.J.Williams, Phys.Rev.45,10 (1934) 729.

[12] D.I.Ivanov e.a. Nucl.Phys. A539 (1992) 263.

[13] I.A.Pshenichnov e.a. Eur.Phys.J. A24 (2005) 69.

[14] D.I.Ivanov e.a. Rus.J.Nucl.Phys.55,10 (1992) 262 .

[15] A.I.L'vov e.a. rus.J.Nucl.Phys.55, 1 (1992) 3.

[16] K.A. Ivanov, S.A. Shulyapov, A.V. Rusakov LPI, Cherenkov readings, (2012) Moscow. 